\title{
Research on Limited Resources Optimal Allocation Model
}

\author{
Tian Yu Guang ${ }^{1 a}$, Ma Liang ${ }^{2 b}$ \\ 1. Department of Surface Ship Command, Dalian Naval Academy, Dalian, 116018, China \\ 2. Department of Surface Ship Command, Dalian Naval Academy, Dalian, 116018,China \\ a446882051@qq.com, b285892287@qq.com
}

\begin{abstract}
Keywords : Optimize configuration; Constraint satisfaction; Spatial coordination; Resource-constrained
\end{abstract}

\begin{abstract}
For formation in collaborative operations conflict caused by the unreasonable resource allocation problems, using the method based on constraint satisfaction, more entities limited resources optimal allocation model is established, and a heuristic algorithm based on rules to solve the model and the simulation calculation, the results show that the method is simple and feasible.
\end{abstract}

\section{Introduction}

Formation cooperative engagement formation is the cooperative combat unit in order to achieve a satisfactory effect and the process of hard work together. In modern naval battle field environment, the goal of the cooperative engagement is not only a coordinated plan execution, more important is to realize collaborative units in space and time consistency of action, so as to achieve the synchronization. Space is an important part of the fleet operational synergy together. In battle time consistency, space synergy is reasonable organization of different area, different direction and different height of combat operations, to achieve mutual close cooperation and support.

Space synergy of the space concept refers to the formation of cooperative combat unit (if no special instructions, use entity instead of unit) physical domain space. Defined as: formation cooperative combat system within any one entity (surface ships, aircraft, or other platforms) or a combination of entities with the same operational purposes according to their own power, the electromagnetic force formed by can effectively control ability and information space. Therefore, the space is the space concept of generalized in this paper.Space cooperation is to adjust the physical space and configuration, make physical space to entities on the consistency of action. Established in this paper, based on constraint satisfaction formation cooperative engagement more entities limited resources optimization allocation model is to solve this problem.

\section{Formation cooperative engagement resources limited decision making based on constraint satisfaction problem model}

Resource-constrained and constraint satisfaction problem. Resource is an important and indispensable component of process management, complete the task in every action (Activity), any entity needs to be on a mission or use a different species, different amount of resources, and the effective availability of these resources tend to have limitations, constitute the constraints of process management, such a problem is known as the problem of limited resources.

Since 1974 Montanari first proposed the constraint satisfaction problems in image processing ${ }^{[1]}$ (constraint satisfaction problems, referred to as the CSPs), constraint satisfaction as an important method in artificial intelligence and computer science and other fields are widely used in a lot of problems, from the queen, a classic problem such as figure dyeing to scheduling, planning, resource allocation and other large application problems, can form into a constraint satisfaction problem solving.

Constraint satisfaction problem is within a certain range for all variables meet the constraint 
relationship between each other assignment problem, the range of values of the variables, and constraints between variables. Resource-constrained problem solving usually USES the planning model, methods such as scheduling and allocation, the algorithm of model with heuristic algorithm and genetic algorithm, ant colony algorithm, etc.

Formation cooperative engagement decision problem model. Formation cooperative engagement is to combat Mission, Mission task) as the center, the wide-area distributed, dynamic deployment of combat system (entity) real-time interaction, integration, efficient overall combat capability. In the study of spatial coordination, only formation cooperative engagement decision-making problem is the entity in the task under the action of spatial configuration.

Will as a combat entity space resources, then a physical space in the form, and will be affected by a variety of spatial ability, pass the process and time limit: (1) the space constraints, the different period of time physical space is not the same. (2) the space by the physical ability limit, different physical space is not the same. (3) the space between the different entities in the mutual restriction, space collision conditions may lead to serious conflict. Therefore, formation cooperative engagement decision problem can be thought of as limited resources, constraint satisfaction model of decision making. The formalization can speak formation cooperative engagement decision problem model is defined as a quad $\mathrm{DQ}=(\mathrm{TE}, \mathrm{TA}, \mathrm{TR}$ and $\mathrm{TC})$.

Among them:

(1) the DQ (decision question), said the decision problem model.

(2) TE (task entity), the task entity, said entity collection of combat missions.

(3) TA (task activity), the task action, said the collection of a series of coordinated combat unit in each action corresponding to the corresponding entities.

(4) the TR (task resourse), the task resources, resource collection, here only refers to the time and space.

(5) TC (task constraint), the task constraints, constraint relationship.

According to formation cooperative engagement decision problem model can more clearly know, based on constraint satisfaction formation to operate with limited resources optimal allocation model is to solve the problem of formation cooperative combat decision. The specific train of thought is: solving satisfy the constraints of physical space in the optimal allocation of tasks in action.

\section{Multiple entities limited resources optimization configuration based on constraint satisfaction problems and model}

Multiple entities limited resources optimization configuration based on constraint satisfaction problem. According to formation cooperative engagement decision problem and resource-constrained project scheduling problem model ${ }^{[2]}$ can be determined based on constraint satisfaction formation cooperative engagement more entities limited resource configuration optimization problem (multi the entity resource constrainted optimization configuration problem, MERCOCP) model.

Formation based on constraint satisfaction to operate with more limited entity resource optimal allocation problem can be simply described as follows: formation cooperative multitasking is the coordination of by the formation of multiple entities to complete combat system, and a series of single entity in multiple entities on the multiple action time of temporal relations, each entity has its own resources and sharing resources, each operation for a certain amount of time, this problem is mainly to solve the timing relationships more entities in the time and resource constraints based on Shared resource optimize configuration, to as to achieve the maximum of formation cooperative combat effectiveness. Question assumptions as follows:

(1) single entity action time of temporal relations have been adjusted for consistency.

(2) each entity, an independent possession of own resources (such as the number of ammunition weapon system, etc.), this has not affect collaborative operational effectiveness. 
(3) the space as the only Shared resource optimize configuration, and space resources.

(4) constraints as the time and space constraints.

More entities limited resources optimization allocation model. According to the operational coordination and control model ${ }^{[3]}$ and more entities optimized configuration of resource constrained mode, based on constraint satisfaction can be determined the entity limited resources optimization allocation model.

Model assumption:

(1) formation cooperative engagement system entities, each entity $E_{i}(i \in[1, n])$ has $m_{i}$ tasks.

(2) the time scale $t_{i j}\left(j \in\left[1, m_{i}\right]\right)$ for each task action, take the task action represent the upper limit of time variable interval.

(3) the time of each task action variable interval value of length $p_{i j}$, showed that physical action task need time.

(4) the entity $E_{i}$ action of tight task $j$ before the first task for $P_{i j}$, said that the task of action $j$ and the first task in time constraints are before or meets relations.

(5) the decision variables $x_{i j}$ of that entity $E_{i}$ in the first task $j$ in action space, $x_{i j}$ values are defined as $\{0,1\}$, indicates that $x_{i j}=1$ the entity $E_{i}$ space of existence in the first task $j$ in action, carried out in accordance with the physical space value to the real space variable assignment, assign a domain is available, $x_{i j}=0$, which indicates that the space of does not exist, the entity space without assignment, otherwise there is a conflict.

(6) if the entity space in action, its operational effectiveness in action as a fixed value $w_{i j}$, this value is obtained through expert consultation, also can be obtained through calculation of the real operational effectiveness.

(7) $E_{i}$ of an entity set with the $j$ task in action space resources possession $S O S_{i j}$.

Thus can build based on constraint satisfaction formation cooperative engagement more entities limited resources optimization allocation model.

The objective function:

$\max z=\sum_{i=1}^{n} \sum_{j=1}^{m_{i}} w_{i j} \cdot x_{i j}$

Constraints:

$$
\begin{aligned}
& \text { (1) } \sum_{j=1}^{m_{i}} x_{i j}=1, \forall i \in[1, n] \\
& \text { (2) } x_{i j} \in\{0,1\} \\
& \text { (3) } \sum_{j=1}^{m_{i}}\left(t_{i j}-p_{i j}\right) \cdot x_{i j}-\sum_{h=1}^{m_{i}} t_{i h} \cdot x_{i h} \geq 0, \forall h \in P_{i j} \\
& \text { (4) }\left(\operatorname{SOS}_{i j} \cdot x_{i j}\right) \cap\left(S O S_{l k} \cdot x_{l k}\right)=0, \forall l \in[1, n], \forall k \in\left[1, m_{l}\right]
\end{aligned}
$$

Constraints (1) indicates that a task must be continuous to complete action has started, the space cannot be preempted. Constraints (2) shows that the decision variables of the feasible region. Constraints (3) shows that single entity task schedule meet the requirement of consistency, and that a task action must act before all its tight task is completed to start. Constraints (4) suggests that space resource constraints, refers to the space of any two entities have the intersection of 0 . 
The heuristic algorithm to solve MERCOCP model based on rules. Now, for this kind of NP-hard problem, swarm intelligence research fields produced two optimization algorithms: ant colony optimization algorithm (ant colony optimization, the ACO) and particle swarm algorithm, particle swarm optimization, PSO). In reference [2] in [4] respectively provides such a model of the $\mathrm{ACO}$ and PSO algorithm. When many simply require a more reasonable solution, can use heuristic algorithm to solve it.

(1) problem solving MERCOCP heuristic rules

Rule one: the goal of MERCOCP problem is to operate with greater effectiveness formation, therefore, in the line of duty as much as possible the arrangement of the entity to perform a task.

Rule 2: to different entities, the consistency problem of time because there is no plan, so you should as far as possible using parallel arrangement of different entities to perform a task.

Rule 3: when the entity space there is a conflict, if is the same type task, should remain operational effectiveness value of physical action, physical action to remove the efficiency value is small, to avoid any conflict.

Rule 4: when an entity of a certain action with multiple entities have conflict, should remove the entity's actions, keep multiple entities, in order to avoid conflict

(2) heuristic algorithm design

(1) according to the real action sequence relational tables one and two cooperative engagement plan according to the rules, all entities action space configuration 1.

(2) according to the results of physical action space collision detection (1) entities in the action whether there is a conflict, if conflict does not exist, go to (5). Output more entities limited resources optimal allocation scheme. If there is a conflict to (3).

(3) according to the rules of the four removal with multiple entities action entity in the conflict.

(4) according to the rules of removing conflict low efficiency value of the three entities.

(5) end of the configuration, the output configuration results.

(3) based on the rules of the heuristic algorithm for example calculation

Hypothesis formation in collaborative operations have three entity, the entity Numbers for 1, 2, 3. Entity 1 need to perform tasks set for action, action number for the 3 . Entity 2 need to perform tasks set for action, action number is 5. Entity 3 need to perform tasks set for action $m_{3}$, action number 4 . For each entity, its task actions have adjusted their consistency. For three entities of a total of 12 action shall be carried out in accordance with the order of a label $\{0,1,2, \cdots, 13\}$, including 0 and 13 for action. Hypothesis according to the operational plan and rules can determine the action sequence as shown in table 1.

Table 1 physical action temporal relational tables

\begin{tabular}{|c|c|c|c|}
\hline Action code & Tight before action & Action code & Tight before action \\
\hline 0 & - & 7 & 6 \\
\hline 1 & 0 & 8 & 3,7 \\
\hline 2 & 1 & 9 & 1 \\
\hline 3 & 2 & 10 & 4,9 \\
\hline 4 & 2 & 11 & 7,11 \\
\hline 5 & 2,4 & 12 & - \\
\hline 6 & 3,5 & 13 & - \\
\hline
\end{tabular}

Other known information slightly. Can work out the calculation result is shown in figure 1. 


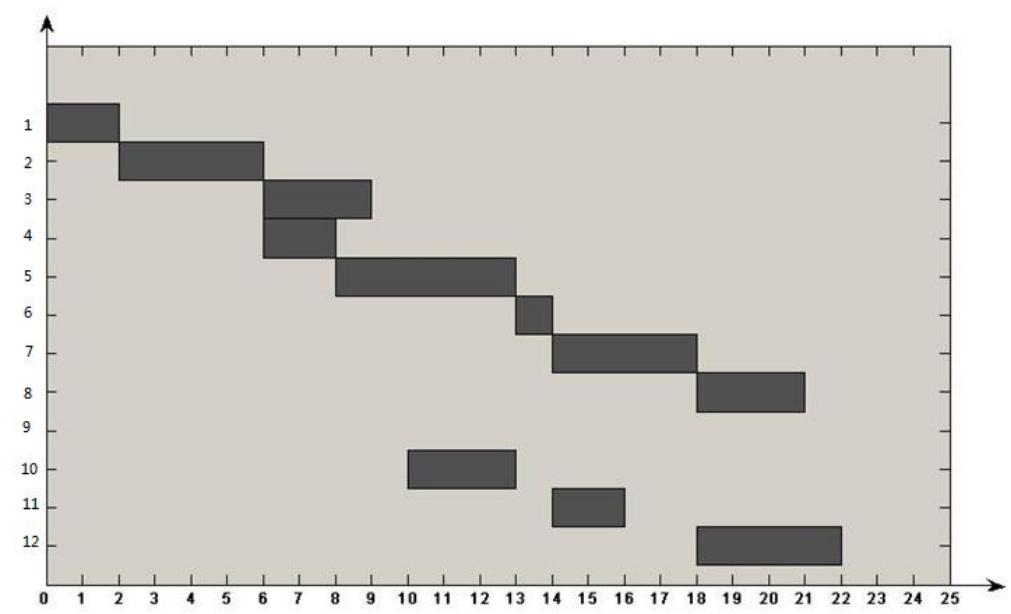

Figure. 1 more entities limited resources optimal allocation results

As shown in figure 1, the order of time, the ordinate said physical action. Figure in the result not only shows the entity space configuration of the action, also gives the start time of action, action time length, the priority sequence of action. Calculation results show that the entity 3 action of 9 can't space configuration.

\section{Conclusion}

Study of complex combat system is the problem in the scientific research workers. This paper adopts the model of constraint problem and the problem of limited resources, building the mode of formation cooperative engagement decision problem and according to the resource-constrained project scheduling problem more pattern to further determine the formation based on constraint satisfaction together more entities optimized configuration of resource model, finally more entities limited resources optimal allocation model is established, the space resource optimal allocation of formation cooperative engagement was studied, calculation results are given. The research conclusion will provide decision-making basis for the establishment of cooperative engagement plan.

\section{References}

[1]Galay Barbarosoglu, Linet Ozdamar, Ahmel Cevik.An Interactive Appmach of Hierarchical Analysis of Helicopter Logistics in Disaster Relief Operations[J]. European Journal of Operational Research, 2002: 118-133

[2]WANG Hong-jun , CHI Zhong-xian.Shipboard hard and soft weapon anti-missile decision-makingoptimization based on collaboration[J], Control and Decision, 2007, 22(3): 299-303

[3]Liang Bo, Duan Ran.Fire distribution model based on feasible direction algorithm[J], Command Information System and Technology, 2013,4（2）:30-32

[4]L Ozdamar. Emergency logistics planning in natural disasters[J]. Annal of Operation Research, 2004, 129(11): 218-219.

[5]Sarit Kraus.Negotiation and cooperation in mulfi-ageat environments.Artificial Intelligence. 1997: 79-97

[6]HUANG Haixin, WANG Ding wei.English auction dynamic model based on fuzzy game[J], Information and Control,2012,41(4):509-513

[7]GAO Zhi hua,CHEN Jian,WEN Jianguo.Optimal Firing Distribution of the Air Defense 
Based on Genetic Algorithm[J],Computer Digital Engineering,2013,44（5）:733-735

[8]Shao Qiu feng, Ma ya long, hu jin chuan.Applied research for fuzzy composite operators in operational alternatives integrated evaluation[J],Journal of Academy of Armored Force Engineering, 2004, 18 (12): 41-43

[9]LI Zi fen, LI Xiang min, CHEN Jin zhu.Dynamic joint fire distribution method based on decentralized cooperative auction algorithm[J],Fire Control \& Command Control, 2012,37( 11 ):50-52

[10]NIAN Song lei, YAN Jian gang, CHEN Rong.The optimization method of antiship missile fire distribution based on dynamic programming[J],Ship Science And Technology, 2012,34 (7) :110-113

[11]MA Liang, QING Zuo-sheng, ZHANG Lin.Formation comprehensive cooperative air defense multi-stage decision optimization modeling[J], Tactical Missile Technology, 2013,9 (5):25-28

[12]LIU Wei dong, JIANG Qing shan, LI Yong.Fire distribution of the network centric ship-to-air missile based on earlier damage[J],Ship Science And Technology, 2011,33 (2):98-101

[13]CHEN Guo sheng, JIA Zi ying.Research on coordinated air defense firepower distribution model for warships[J], Command ControI\& Simulation, 2011,33 (12):13-15 